\title{
Influence of adverse weather on drivers' perceived risk during car following based on driving simulations
}

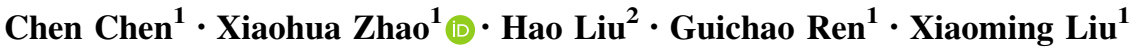

Received: 31 October 2018/Revised: 31 July 2019/Accepted: 19 August 2019/Published online: 19 September 2019

(C) The Author(s) 2019

\begin{abstract}
Adverse weather has a considerable impact on the behavior of drivers, which puts vehicles and drivers in hazardous situations that can easily cause traffic accidents. This research examines how drivers' perceived risk changes during car following under different adverse weather conditions by using driving simulation experiment. An expressway road scenario was built in a driving simulator. Eleven types of weather conditions, including clear sky, four levels of fog, four levels of rain and two levels of snow, were designed. Furthermore, to simulate the carfollowing behavior, three car-following situations were designed according to the motion of the lead car. Seven car-following indicators were extracted based on risk homeostasis theory. Then, the entropy weight method was used to integrate the selected indicators into an index to represent the drivers' perceived risk. Multiple linear regression was applied to measure the influence of adverse weather conditions on perceived risk, and the coefficients
\end{abstract}

Xiaohua Zhao

xiaohuazhao618@gmail.com

Chen Chen

chen-chen@emails.bjut.edu.cn

Hao Liu

hao.liu@bjjtw.gov.cn

Guichao Ren

rengc@emails.bjut.edu.cn

Xiaoming Liu

liuxm@bjut.edu.cn

1 Beijing Engineering Research Center of Urban Transport Operation Guarantee, College of Metropolitan

Transportation, Beijing University of Technology, Beijing 100124, China

2 Beijing Transportation Information, Beijing 100161, China were considered as indicators. The results demonstrate that both the weather conditions and road type have significant effects on car-following behavior. Drivers' perceived risk tends to increase with the worsening weather conditions. Under conditions of extremely poor visibility, such as heavy dense fog, the measured drivers' perceived risk is low due to the difficulties in vehicle operation and limited visibility.

Keywords Adverse weather - Driving simulation . Perceived risk $\cdot$ Entropy weight method $\cdot$ Multiple linear regression

\section{Introduction}

Driving performance changes when there is a variation in the external environment. One of considerable factors that affect driving performance is the weather condition, such as fog, rain and snow. These weather conditions are associated with visibility as well as the friction of the road surface and further influence driving safety.

The parameters related to vehicle motion, such as acceleration and headway time, can be used to measure how drivers respond to the various driving conditions. Many previous studies have analyzed driver behavior under the influence of weather. Rahman and Lownes [1] studied the impact of three weather conditions, no rain, light rain and moderate rain, on car-following behavior by using the time gap, following distance and vehicle speed as indicators. The analysis indicated that rainy conditions caused a greater time gap and spread in speed distributions than dry 
conditions did, and no statistically significant difference was found in following distance. Billot et al. [2] analyzed the effects of three types of weather conditions on driving behavior. The results suggested that the percentage of headways less than $2 \mathrm{~s}$ dropped by $18 \%$ and the percentage of spacings less than $50 \mathrm{~m}$ decreased $20 \%$ on rainy days. Saberi and Bertini [3] considered the effects of four levels of rain intensity, very light rain, light rain, moderate rain and heavy rain, on the maximum speed. The results indicated a decrease in speed with increasing rain intensity. However, in their study, the samples in heavy rain were not sufficient to draw meaningful conclusions. The abovementioned studies are based on field-collected data with the common problem that extreme weather conditions are rarely considered due to the uncontrollable nature of weather. Furthermore, weather also influences the traffic condition, such as traffic volume, which also changes driving behavior. These two problems complicate the integrated analysis of driving behaviors under different adverse weathers.

Driving simulation provides another method of analyzing the influence of weather on driving behavior, as both weather conditions and traffic environment can be well controlled. Yan et al. [4] designed three levels of fog, no fog, light fog and heavy fog, in a driving simulator and found that drivers would reduce their driving speeds and acceleration to lower driving risk. Konstantopoulos et al. [5] designed rainy weather conditions in a driving simulator and found that rainy weather had a significant effect on the eye movement of drivers. Broughton et al. [6] used a driving simulator to analyze the influence of visibility on car-following behavior. The experiments indicated that drivers were separated into two groups in foggy weather: staying within and lagging beyond the visible range of the lead car. Yamaguchi and Sakakima [7] used a driving simulator to analyze the effect of a snow-covered road on driving behavior. These studies have demonstrated the effectiveness of driving simulators in simulating different weather conditions and supporting driving behavior analysis.

Due to the considerable impacts of weather on driving behavior, the occurrence of adverse weather always puts vehicles and drivers in hazardous situations and increases the risk of traffic accidents. Although many researchers have found driving behavior changes under adverse weather conditions, how drivers perceive and react to environment changes, i.e., the risk perception, remains unclear.

Risk perception is a concept that describes how a driver recognizes the hazard level of the driving environment and is used to explain why and how drivers adjust their behavior when facing inclement weather. Risk perception is a subjective concept and is typically measured by questionnaires. Rhodes and Kelly [8] analyzed the influence of age and gender on risky driving by a phone survey. DeJoy [9] used self-reported data to measure the gender difference in traffic accident risk perception. However, different levels of risk perception will result in different driving behaviors and vehicle motions, which can in turn be used to measure the risk perception level of the drivers. In Bella's research [10], the effects of roadside configurations on risk perception were analyzed with vehicle speed and lateral position as indicators. Hjelkrem and Ryeng [11] analyzed the perceived risk of drivers under different levels of rain and snow based on field data. Two earlier researchers used a risk compensation hypothesis [12] and risk homeostasis theory [13], respectively. The two theories are similar in that drivers dynamically adjust their driving operations according to the continuous comparison between the perceived risk level and target level of risk (see Fig. 1). The target risk level is the level that individuals are willing to take according to the realtime driving environment, such as the road surface friction or road alignment. Individuals generally believe that they will not be trapped in an accident if the risk level that they perceive is lower than or equal to the target level. In contrast, if drivers perceive a risk level higher than the target level, actions will be taken to decrease the risk level. For example, drivers will decrease their speeds in a carfollowing situation to avoid a rear-end collision when the lead car is decelerating.

According to the risk homeostasis theory, we propose to measure the risk perception level of drivers under different weather conditions by utilizing vehicle motion parameters. Although similar research has been conducted by Hjelkrem and Ryeng [11], the previous research has a limitation in field data; namely, few observations were made under moderate/heavy rain/snow.

This paper is structured as follows: Sect. 2 introduces the methodology of this paper, including the apparatus, experiment design and indicator selection. The results are presented in Sect. 3 and then discussed in Sect. 4. Finally, conclusions are shown in Sect. 5.

\section{Methodology}

\subsection{Apparatus}

This research used a fixed-base driving simulator located in the Key Laboratory of Traffic Engineering at Beijing University of Technology. The simulator includes a modified car (replacing the original vehicle accessories with computers or dynamic sensors), control computers and video and audio devices. Driving circumstances are projected onto four large screens (three ahead of and one behind the simulator car) and are displayed on two small 


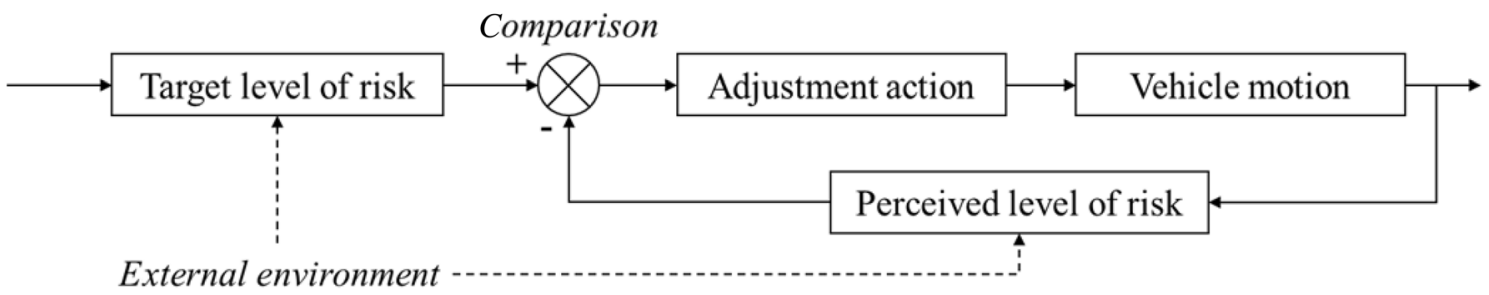

Fig. 1 Risk homeostasis theory
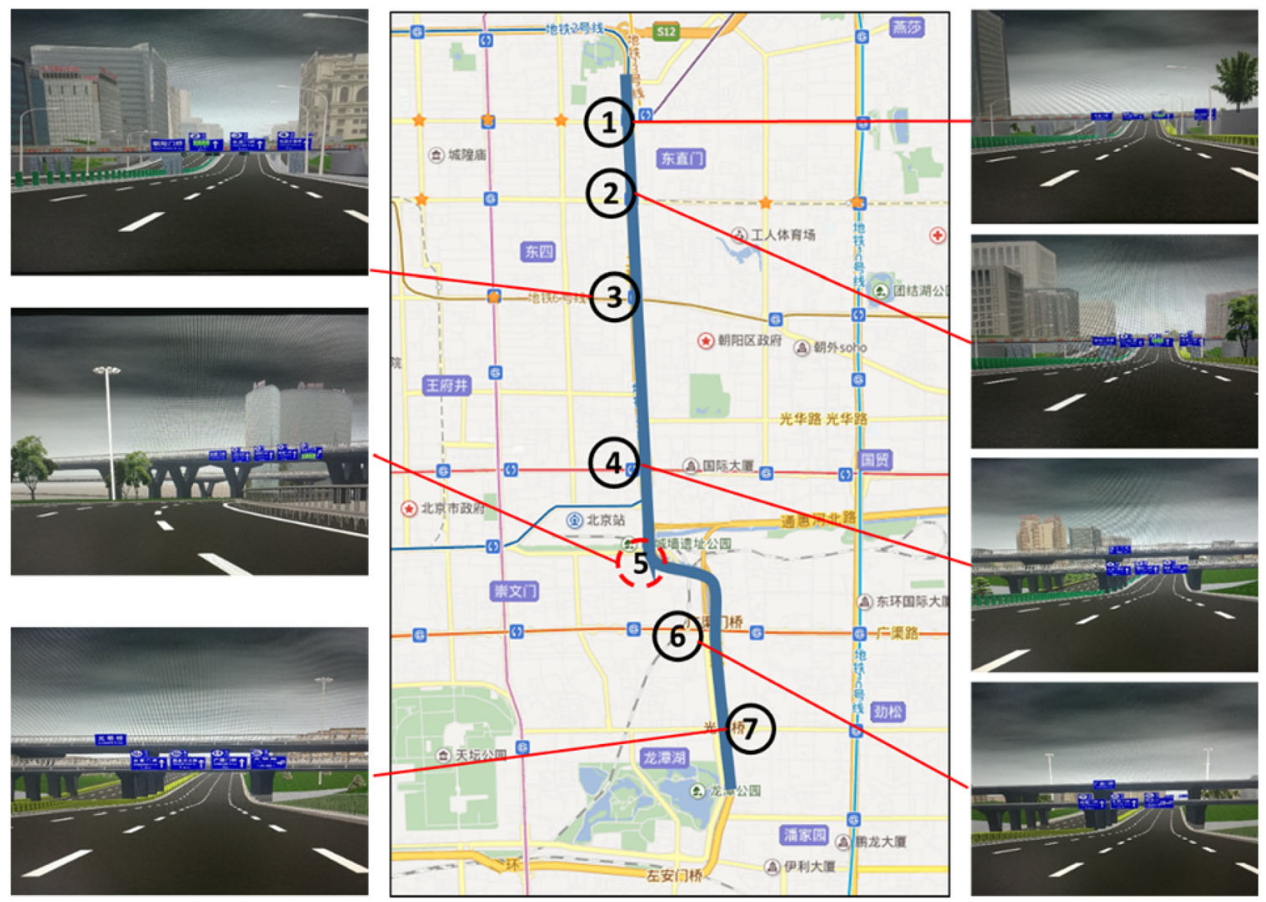

Fig. 2 Overall simulation scenario

screens on both sides of the car as side mirrors. This simulator can record driver behavior responses (e.g., gas pedal, brake pedal, steering wheel angle, and direction indicator state) and vehicle operation data (e.g., speed, acceleration, distance to lead/rear car, $X / Y$ coordinates, and lateral shift) at $1-50 \mathrm{~Hz}$.

Previous research has verified the validity of this simulator in studying driving behavior [14, 15]. A total of 250 drivers in the past few years participated in driving simulation experiments, and this simulator was evaluated through questionnaires. The average score about how the driving simulator reflects reality reaches 8 ( 1 for not real at all and 10 for very real).

\subsection{Scenario design}

The design of the scenario is based on Beijing East 2nd Ring Road from Zuoanmen Bridge to Xizhimen Bridge, which is approximately $10 \mathrm{~km}$ long, as shown in Fig. 2. The simulated road has three lanes in each direction, with a width of $4 \mathrm{~m}$ per lane. There are seven interchange bridges (labeled as (1)-(7) in Fig. 2) in the selected range. According to the design parameters obtained from the road management department, 6 of the 7 interchanges (in terms of expressways) have similar structures and road slope parameters. In the simulation scenario, the same parameters $(3 \mathrm{~m}$ height and $2 \times 230 \mathrm{~m}$ length, with the slope of $1.3 \%$ ) are used to model the 6 interchanges (bridges 1, 2, 3, 4, 6, and 7 in Fig. 2). The other interchange is different because it contains both a curve and a horizontal slope (bridge 5 in Fig. 2). The simulated road can be separated into two slopes at the interchange regions: downslope and upslope. The roads between the two interchange bridges are regarded as a basic segment. The road on bridge 5 is not considered because of its complex alignment. Thus, three road types are included in this scenario: basic segment, upslope and downslope. The lengths of the basic segment range from 700 to $1800 \mathrm{~m}$, and the slope length is $230 \mathrm{~m}$ for both upslope and downslope. 


\subsection{Weather design}

In this research, 11 weather conditions were designed by configuring three parameters in driving simulation software: SetRain, SetSnow, and SetFog. By changing the values of SetRain and SetSnow, the projected screens display the visual effects with different levels of rain or snow intensity. The SetFog (ranging from 0 to $10,000 \mathrm{~m}$ ) is used to control the visibility distance.

SetRain and SetSnow are in percentage rather than in the precipitation unit $(\mathrm{mm} / 12 \mathrm{~h})$. The values 0 and $1(100 \%)$ present two extreme cases: 0 stands for no rain/snow, and 1 for the heaviest rain/snow. Additionally, the road friction coefficient (in \%) that is a friction reduction coefficient comparative to the one in sunny days cannot be modified because it is set automatically by the driving simulation software according to the value of SetRain or SetSnow. The friction parameter has four levels $(100 \%, 75 \%, 60 \%$, or $45 \%)$ which correspond to the ranges of SetRain (0\%-24\%, $25 \%-50 \%, 21 \%-74 \%$, and $75 \%-100 \%$, respectively) and has two levels (45\% or $20 \%$ ) which correspond to the ranges of SetSnow (0\%-50\% and 51\%-100\%, respectively). Eleven weather conditions (clear sky and ten types of adverse weather conditions) were configured in the driving simulator, and their parameters are shown in Table 1. Four/two levels of rain/snow conditions were designed due to the road friction partitions that are preset by the driving simulation software. The four levels of fog come from the provisions of the national standard [16]. The default setting in driving simulator is clear sky and its visibility distance is set as $10,000 \mathrm{~m}$; i.e., the fog has no effect on drivers.

A static weather matching experiment was conducted with 30 student participants to establish the relationship between the driving simulation weather environment and actual weather grade used in the weather grading in China
[16-18]. Students chose the most similar actual weather grade (the definitions of the grades of actual weather has been informed to these students) for every simulated weather condition after observing 11 weather scenarios on screens in a random order. The matching results are shown in Table 1. The visual effects of certain weather conditions are shown in Fig. 3.

\subsection{Car-following design}

Referring to the actual car-following situation, three simplified but typical car-following situations were designed according to the operating states of the lead car: cruising, accelerating and decelerating. In a simulation scenario, the lead car is automatically controlled by the driving simulation software. The lead car typically cruises at $40 \mathrm{~km} / \mathrm{h}$, and participants must follow the lead car. When the following vehicle passes certain locations, the speed trajectories of the lead car under these three conditions are shown in Fig. 4.

The parameters were determined by repetitive adjustments and tests and were thus considered to allow drivers to have nearly real car-following experiences. In the following analysis, the data obtained during a $5 \mathrm{~s}$ period are used.

The three car-following situations are uniformly and randomly allocated on different road types (basic segment, upslope and downslope). When approaching the designated locations, the lead car will perform the designated motions.

\subsection{Experimental procedure}

Thirty-one paid participants ( 24 males and 7 females, age $30 \pm 7.9$ years) were recruited for this research. All the participants are employees of a driving service company with professional driving skills (driving age of

Table 1 Configurations of the 11 weather conditions

\begin{tabular}{|c|c|c|c|c|c|c|}
\hline \multirow[t]{2}{*}{ ID } & \multicolumn{4}{|c|}{ Configuration of three functions } & \multirow[t]{2}{*}{ Matched weather condition (actual weather grade) } & \multirow[t]{2}{*}{ Abbreviation } \\
\hline & SetRain $(\%)$ & SetSnow $(\%)$ & $\operatorname{SetFog}(\mathrm{m})$ & Friction $(\%)$ & & \\
\hline 1 & - & - & 10,000 & 100 & Clear sky & $\mathrm{CS}$ \\
\hline 2 & - & - & 1500 & 100 & Light fog $(1000 \mathrm{~m}<S \leq 100,000 \mathrm{~m})$ & $\mathrm{LF}$ \\
\hline 3 & - & - & 800 & 100 & Fog $(500 \mathrm{~m}<S \leq 1000 \mathrm{~m})$ & $\mathrm{F}$ \\
\hline 4 & - & - & 300 & 100 & Dense fog $(200 \mathrm{~m}<S \leq 500 \mathrm{~m})$ & DF \\
\hline 5 & - & - & 50 & 100 & Heavy dense fog $(50 \mathrm{~m}<S \leq 200 \mathrm{~m})$ & $\mathrm{HDF}$ \\
\hline 6 & 20 & - & 2000 & 100 & Light rain $(0-9.9) \mathrm{mm} / 24 \mathrm{~h}$ & LR \\
\hline 7 & 45 & - & 800 & 75 & Rain (10.0-24.9) mm/24 h & $\mathrm{R}$ \\
\hline 8 & 70 & - & 550 & 60 & Heavy rain $(25.0-49.9) \mathrm{mm} / 24 \mathrm{~h}$ & HR \\
\hline 9 & 95 & - & 300 & 45 & Extremely heavy rain $(100.0-249.0) \mathrm{mm} / 24 \mathrm{~h}$ & EHR \\
\hline 10 & - & 45 & 500 & 45 & Snow $(2.5-4.9) \mathrm{mm} / 24 \mathrm{~h}$ & S \\
\hline 11 & - & 95 & 100 & 20 & Extremely heavy snow (10-19.9) $\mathrm{mm} / 24 \mathrm{~h}$ & EHS \\
\hline
\end{tabular}

$S$ denotes visibility distance 


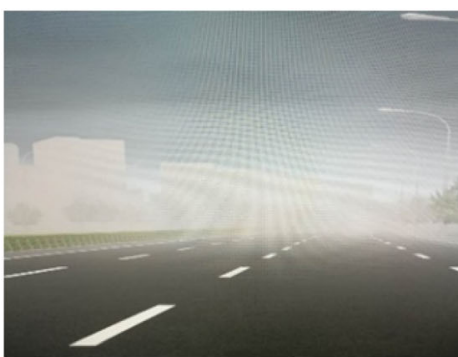

(a) Dense fog

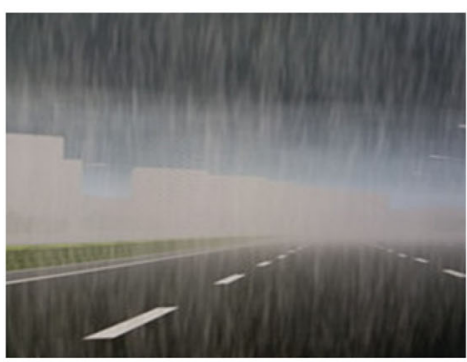

(d)Extremely heavy Rain

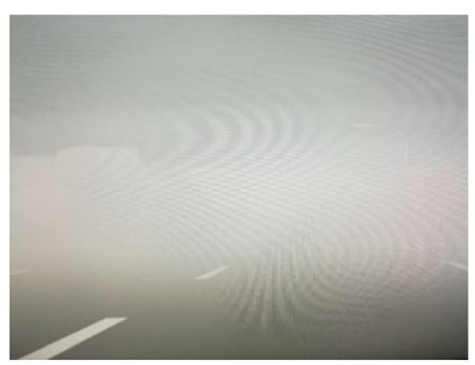

(b) Heavy dense fog

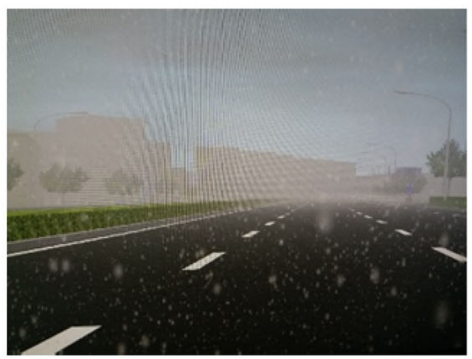

(e) Snow

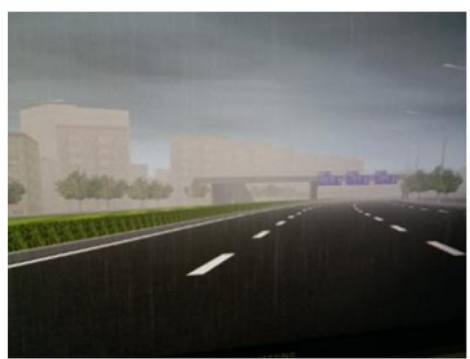

(c) Rain

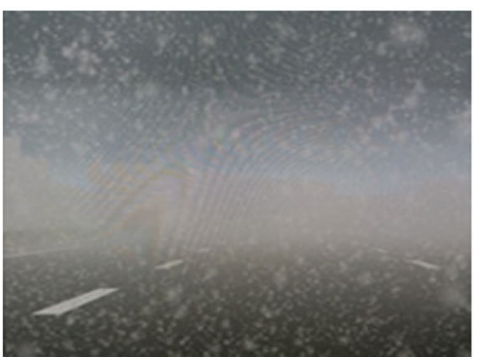

(f) Extremely heavy snow

Fig. 3 Visual effects of the simulated weather

The lead car's motion

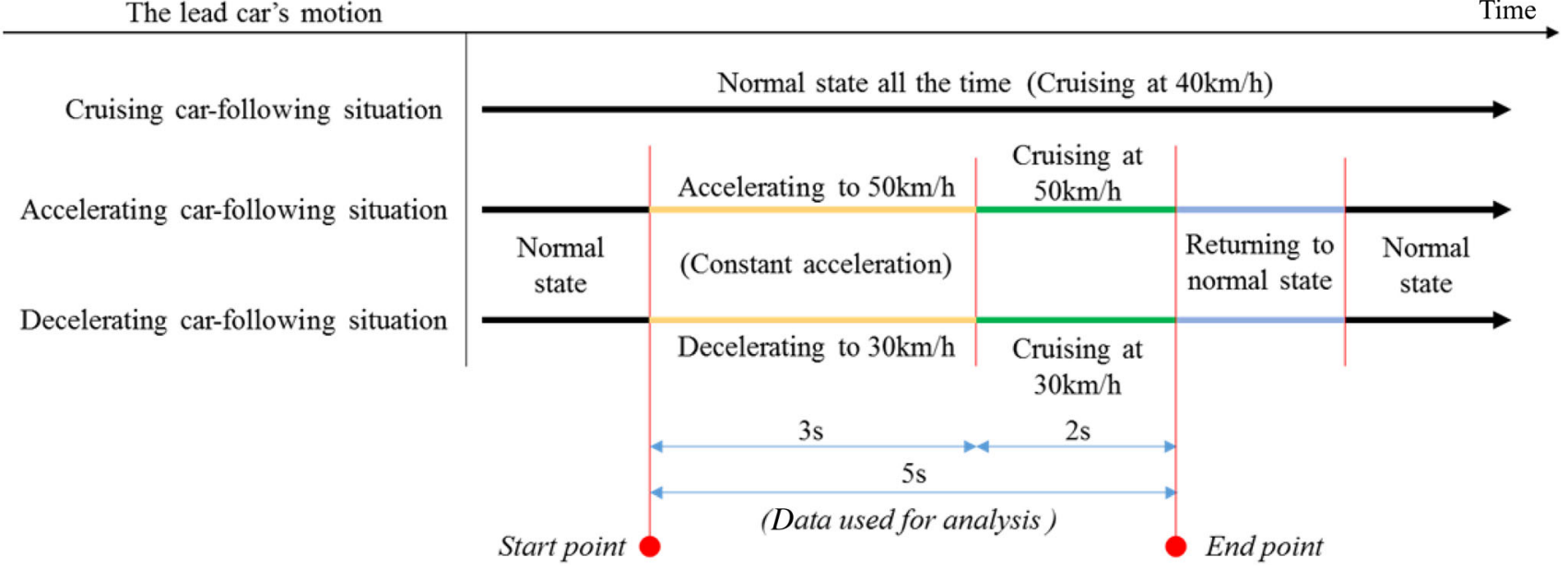

Fig. 4 Motion of the lead car

$15.8 \pm 6.9$ years) and driving experiences with multiple types of vehicles.

Once a driver arrives at the driving simulation laboratory, a pre-driving tutorial that lasts for approximately 10 min must be performed to help the driver adapt to the experiment equipment. Then, the participant was asked to complete a questionnaire about basic information, including age, age of driving, time to fall asleep, time to wake up, and whether medication or alcohol is taken. If a driver slept less than $6 \mathrm{~h}$ or took medicine or alcohol during the past $12 \mathrm{~h}$, his/her experiment would be cancelled and adjusted to a later time.
For each driver, 11 different scenarios are designed with 11 different weather conditions. Coupled with road type and car-following situation, an $11 \times 3 \times 3$ (weather condition $\times$ road type $\times$ car-following situation) designed experiment is performed. During the driving experiments, participants were asked to follow the lead car. The average driving time for each scenario is approximately $7 \mathrm{~min}$. The overall experiment time for each driver is approximately 90 min, including several rest periods between two scenarios. Driving behavior (e.g., gas pedal, brake pedal, and steering wheel) and vehicle operation data (e.g., speed, 
acceleration and distance to the lead car) were recorded at $20 \mathrm{~Hz}$ concurrently during the driving simulation.

\subsection{Indicator selection}

This paper focuses on measuring the drivers' perceived risk level under different weather conditions. As introduced earlier, several vehicle operation parameters are chosen and then integrated into one index to represent the perceived risk of the drivers. Then, multiple linear regression is conducted, and the coefficients are used to measure the influence of weather conditions and road type on the drivers' perceived risk.

As described in the introduction, several vehicle motion parameters are selected as indicators, including headway distance and acceleration. A traditional indicator, time-tocollision (TTC), is not used in this paper because the lead car has a stable speed, which makes the distance between the lead car and the following car have a similar function as TTC. In addition, in the case of the road surface with reduced friction, the drivers focus more on the vehicle speed control because both sharp acceleration and deceleration have potential hazards. These chosen parameters can describe driver manipulation reacting to the lead car's motion and reflect drivers' perceived risk levels. To better depict driver reaction in different car-following situations, these parameters are selected with different forms. The selected indicators are listed in Table 2 , and their relationships with perceived risk are also provided.

The selected indicators are denoted as $X, Y$ and $Z$ according to the following rules: $X$ represents the car-following situation; $Y$ represents the parameter; and $Z$ represents the statistical measure used to deduce the indicators with the parameters.

The different values (PA and NA) of the acceleration parameters are used according to the car-following situations. Three different indicators, C.H.Ave, A.H.Max and D.H.Min, are deduced from the headways with different statistical methods for three car-following situations:

- For the acceleration car-following situation, the maximum headway indicates the response of drivers to the lead car acceleration (a large maximum headway indicates a low state change of the following vehicle, indicating high cautiousness and a high perceived risk level);

- For the deceleration car-following situation, the minimum headway is more suitable.

- For the cruising car-following situation, the average headway is selected due to the stable speed of the lead car.

When conducting the driving simulation experiments, a non-car-following state is observed, particularly in lowvisibility scenarios, such as heavy dense fog, extremely
Table 2 Selected parameters

\begin{tabular}{|c|c|c|}
\hline Abbreviation & Description & $\begin{array}{l}\text { Relationship with } \\
\text { perceived risk* }\end{array}$ \\
\hline \multicolumn{3}{|c|}{ In the cruising car-following situation (C) } \\
\hline C.H.Ave (m) & Average headway $(\mathrm{H})$ & $\begin{array}{l}\text { C.H.Ave } \uparrow \text {, perceived } \\
\text { risk } \uparrow \\
\text { High perceived risk } \\
\text { makes drivers keep far } \\
\text { away from the lead car } \\
\text { to achieve the target } \\
\text { risk }\end{array}$ \\
\hline $\begin{array}{l}\text { C.PA.Ave } \\
\left(\mathrm{m} / \mathrm{s}^{2}\right)\end{array}$ & $\begin{array}{l}\text { Average (Ave) positive } \\
\text { acceleration (PA) of } \\
\text { the following vehicle }\end{array}$ & $\begin{array}{l}\text { C.PA.Ave } \downarrow \text {, perceived } \\
\quad \text { risk } \uparrow \\
\text { High perceived risk } \\
\text { caused by friction } \\
\text { reduction leads } \\
\text { moderate acceleration }\end{array}$ \\
\hline $\begin{array}{l}\text { C.NA.Ave } \\
\left(\mathrm{m} / \mathrm{s}^{2}\right)\end{array}$ & $\begin{array}{l}\text { Average negative } \\
\text { acceleration (NA) (i.e., } \\
\text { the deceleration value) } \\
\text { of the following } \\
\text { vehicle }\end{array}$ & $\begin{array}{l}\text { IC.NA.Avel } \downarrow \text {, perceived } \\
\quad \text { risk } \uparrow \\
\text { High perceived risk } \\
\text { caused by friction } \\
\text { reduction leads } \\
\text { moderate deceleration }\end{array}$ \\
\hline
\end{tabular}

In the accelerating car-following situation (A)

$\begin{array}{ccc}\begin{array}{c}\text { A.H.Max } \\ (\mathrm{m})\end{array} & \text { Maximum headway } & \begin{array}{c}\text { A.H.Max } \uparrow \text {, perceived } \\ \text { risk } \uparrow\end{array} \\ & & \begin{array}{l}\text { High perceived risk } \\ \text { makes drivers } \\ \text { negatively follow the } \\ \text { lead car to achieve the } \\ \text { target risk }\end{array} \\ & & \begin{array}{l}\text { A.PA.Ave } \downarrow \text {, perceived } \\ \text { risk } \uparrow\end{array} \\ \begin{array}{ccc}\text { A.PA.Ave } \\ \left(\mathrm{m} / \mathrm{s}^{2}\right)\end{array} & \text { Average positive } & \text { High perceived risk } \\ & \text { acceleration of the } & \text { caused by friction } \\ & \text { following vehicle } & \text { reduction leads to } \\ & & \text { moderate acceleration }\end{array}$

In the decelerating car-following situation (D)

D.H.Min (m) Minimum headway
D.H.Min $\uparrow$, perceived risk $\uparrow$
High perceived risk makes drivers positively decelerate to achieve the target risk
ID.NA.Avel $\downarrow$, perceived risk $\uparrow$
High perceived risk caused by friction reduction leads to moderate deceleration
D.NA.Ave Average negative $\left(\mathrm{m} / \mathrm{s}^{2}\right) \quad$ acceleration (i.e., the deceleration value) of the following vehicle

*C.H.Ave $\uparrow$, perceived risk $\uparrow$ ' means 'the increase of C.H.Ave represents that driver perceived risk increases. Other indicators has similar logit relationship

heavy rain and extremely heavy snow. Because the average traffic flow speed is $40 \mathrm{~km} / \mathrm{h}$ and the minimum visibility distance is $50 \mathrm{~m}$ (heavy dense fog), the data with a high 
average headway $(>50 \mathrm{~m})$ are determined to be a non-carfollowing state and are deleted in the dataset.

\section{Results}

In this paper, basic segment and clear sky are regarded as normal conditions. There are two aspects in the analysis on the effect of adverse weathers on traffic flow. First, the influence of weather on each indicator is analyzed. Then, a comprehensive index used to assess drivers' perceived risk is proposed using the entropy weight method and a linear regression model is established to quantitatively evaluate the influence of weather and road types on perceived risk.

\subsection{Influence of weather condition and road type on car-following behavior}

Repeated measures analysis of variance (RANOVA) is used to analyze the influence of two factors (road type and weather conditions) on the values of the selected indicators. The test results are provided in Table 3.

Table 3 shows that both road type and weather conditions have a significant influence on the majority of indicators. However, no significant influence on any of the indicators from the interaction of two factors is observed.

These indicators are classified according to their physical meanings, and their variations under different conditions are plotted in Fig. 5.

In Fig. 5a, the three headway-related indicators increase with the worsening weather conditions. For every indicator, the perceived risk level is the highest under EHS condition.

In Fig. 5a, b, the values of the three headway-involved indicators are obviously related to the operation methods. In Fig. 5c-f, the absolute values of A.PA.Ave and D.NA.Ave are larger than the absolute values of C.PA.Ave and C.NA.Ave, respectively, due to the different lead car motions in the three car-following situations.
Table 3 indicates that weather condition has no significant effect on C.PA.Ave, as can be seen in Fig. 5c. This suggests that the perceived risk when a driver follows a cruising vehicle is not influenced by weather condition. The A.PA.Ave values of adverse weather conditions are lower than that under CS, which indicates that adverse weather conditions will lead to high perceived risk. In addition, A.PA decreases (the perceived risk increases) with a deterioration in the weather conditions for each weather category (rain, snow and fog). The exceptions are in the conditions of extremely adverse weather, such as heavy dense fog and extremely heavy rain, partly because of the significant reduction in visibility when drivers tend to keep up with the lead car in these situations.

In Fig. 5e, C.NA.Ave does not show significant difference for weather conditions, which is in accordance with the results of Table 3. For CS, there is a lowest D.NA.Ave. The absolute D.NA.Ave values in adverse weather conditions correspond to similar A.PA.Ave values, demonstrating that the perceived risk increases in adverse weather conditions. Comparison between absolute values of A.PA.Ave and values of D.NA.Ave indicates that drivers are more cautious toward the speed reduction of lead car than the acceleration of the lead car.

In Fig. 5b, C.H.Ave, A.H.Max and D.H.Min have the highest values on the basic segment. For both A.H.Max and D.H.Min, the values of downslope are higher than of upslope, indicating that drivers are aware of higher risk on a downslope than that on an upslope.

In Fig. 5d, C.PA.Ava and A.PA.Ave have the highest values on downslopes and basic segments, respectively. This result may be attributed to the road slope and different levels of drivers' attentiveness.

In Fig. 5e, the absolute values of C.NA.Ave are smaller on the basic segments than on other road types. There is no significant difference in D.NA.Ave under the different road types.

Table 3 Multivariate test results

\begin{tabular}{|c|c|c|c|c|c|c|}
\hline \multirow[t]{2}{*}{ Indicator name } & \multicolumn{2}{|c|}{ Weather conditions } & \multicolumn{2}{|c|}{ Road type } & \multicolumn{2}{|c|}{ Weather conditions $\times$ road type } \\
\hline & $F_{(10,963)}$ & Sig. & $F_{(2,963)}$ & Sig. & $F_{(32,963)}$ & Sig. \\
\hline C.H.Ave & 3.909 & $0.000 * *$ & 14.808 & $0.000 * *$ & 0.588 & 0.923 \\
\hline C.PA.Ave & 0.681 & 0.743 & 34.897 & $0.000 * *$ & 0.472 & 0.977 \\
\hline C.NA.Ave & 1.016 & 0.428 & 6.764 & $0.001 * *$ & 0.527 & 0.956 \\
\hline A.H.Max & 2.279 & $0.012 * *$ & 11.725 & $0.000 * *$ & 1.290 & 0.176 \\
\hline A.PA.Ave & 3.013 & $0.001 * *$ & 8.539 & $0.000 * *$ & 0.331 & 0.998 \\
\hline D.H.Min & 2.074 & $0.024 * *$ & 3.937 & $0.020 * *$ & 0.489 & 0.971 \\
\hline D.NA.Ave & 6.013 & $0.000 * *$ & 1.607 & 0.201 & 0.662 & 0.865 \\
\hline
\end{tabular}

**Influence is significant at the 0.01 level

*Influence is significant at the 0.05 level 


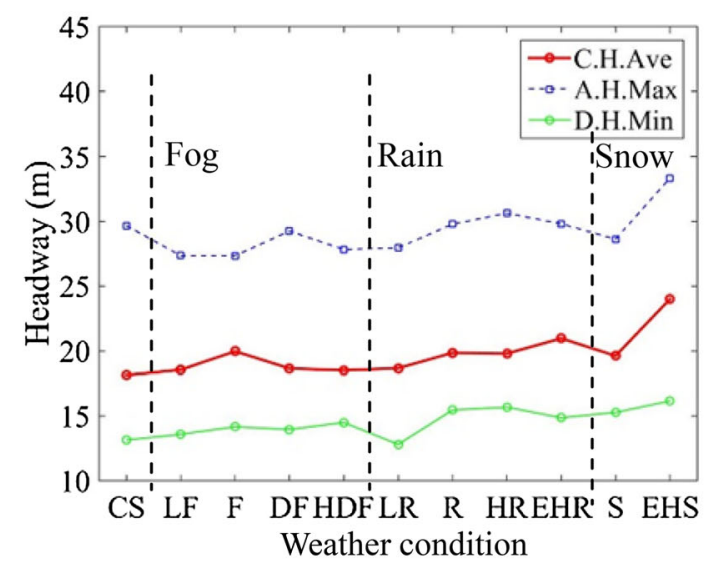

(a) Headway vs weather conditions

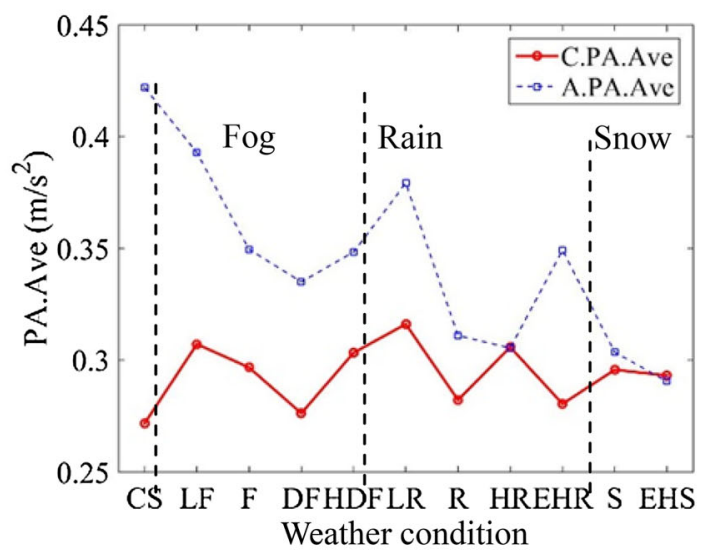

(c) PA.Ave vs weather conditions

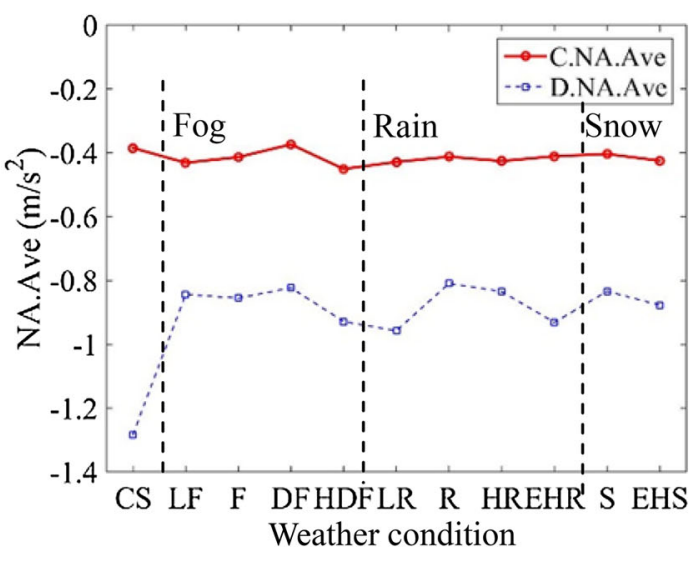

(e) NA.Ave vs weather conditions

Fig. 5 Influence of weather conditions and road types o indicators

\subsection{Influence of weather conditions and road type on the perceived risk of drivers}

In this study, the entropy weight method and multiple linear regression are used to explore the effect of different weather conditions on the perceived risk level of drivers.

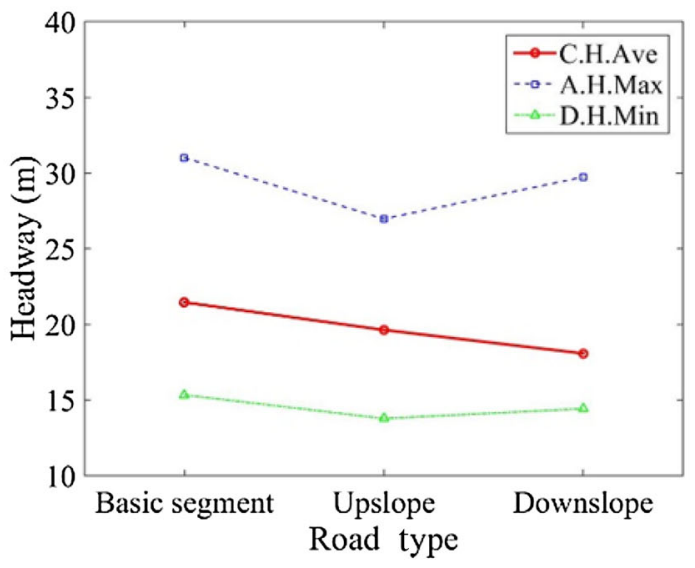

(b) Headway vs road types

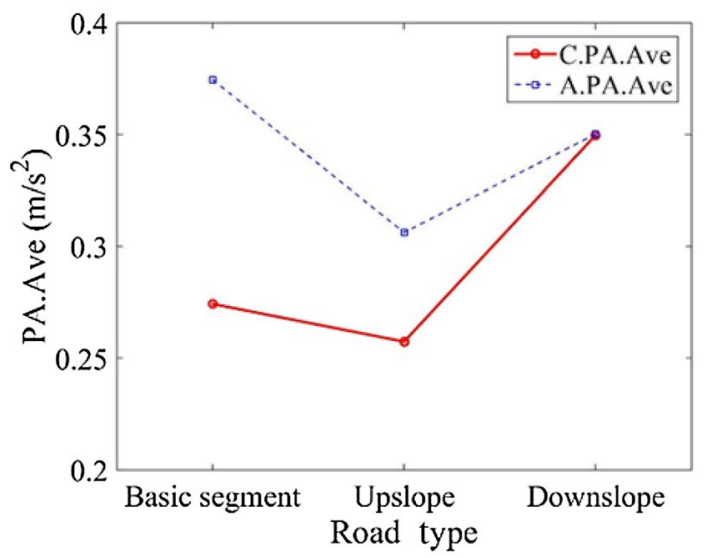

(d) PA.Ave vs road types

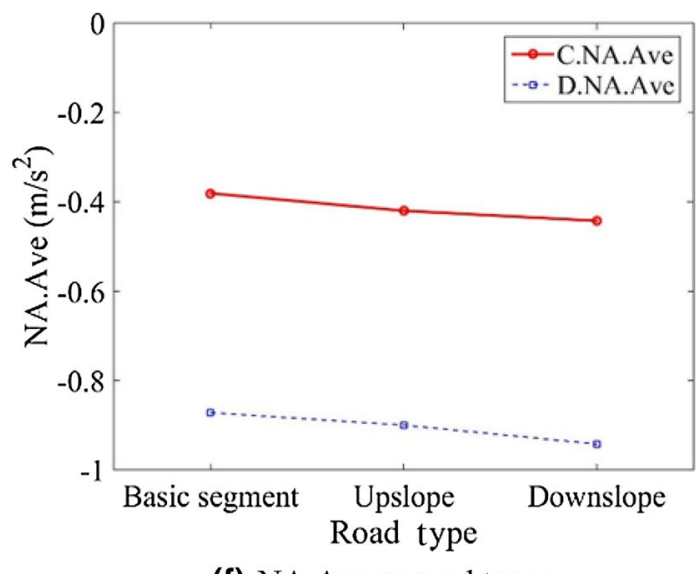

(f) NA.Ave vs road types

The entropy weight method is used to integrate the selected indicators into one index that represents the perceived risk levels of drivers under adverse weather conditions. Then, the multiple linear regression is used to evaluate the influence of weather conditions and road type on the perceived risk level via regression coefficients. 
Before applying the entropy weight method, the selected indicators are normalized to eliminate the influence of dimensionality and ensure the consistency of indicator influence on the perceived risk of drivers.

\subsubsection{Normalization of the original evaluating matrix}

In this work, there are 7 indicators and 33 objects for evaluating (11 weather conditions $\times 3$ road types), which form an original evaluation matrix $\boldsymbol{X}=\left(x_{i j}\right)_{33 \times 7}$. In this matrix, the value of each object is an average of all drivers' data.

Range normalization is used to normalize the indicators. Indicators are classified into two categories, positive or negative indicators, according to their relationship with perceived risk. Positive indicators are those whose values are positively correlated with perceived risk, whereas negative indicators are those whose values are negatively correlated with perceived risk. According to Table 2, C.H.Ave, C.NA.Ave, A.H.Max, D.H.Min and D.NA.Ave are positive indicators, and C.PA.Ave and A.PA.Ave are negative indicators. The normalization algorithms for positive and negative indicators are presented in Eqs. (1) and (2):

$y_{i j}^{\mathrm{p}}=\frac{x_{i j}-\operatorname{Min} x_{j}}{\operatorname{Max} x_{j}-\operatorname{Min} x_{j}}$,

$y_{i j}^{\mathrm{n}}=\frac{\operatorname{Max} x_{j}-x_{i j}}{\operatorname{Max} x_{j}-\operatorname{Min} x_{j}}$,

where $y_{i j}^{\mathrm{p}}$ is normalized positive indicator; $y_{i j}^{\mathrm{n}}$ is normalized negative indicator; $x_{i j}$ is the value of the $i$ th evaluating object of the $j$ th indicator; Min $x_{j}$ is the minimum value of the $j$ th indicator, and $\operatorname{Max} x_{j}$ is the maximum value of the $j$ th indicator.

Equations (1) and (2) are used to obtain the normalized matrix $\boldsymbol{Y}=\left(y_{i j}\right)_{33 \times 7}, y_{i j} \in[0,1]$. All the indicators are positively correlated with the perceived risk.

\subsubsection{Entropy weight method}

According to the information theory, high entropy denotes small variations for the indicator, which indicates that this indicator provides less information. Thus, the indicator has less weight in the integrated index. The entropy of the $j$ th indicator is defined as

$H_{j}=-k \sum_{i=1}^{33} f_{i j} \ln f_{i j}, \quad i=1,2, \ldots, 33$,

where $f_{i j}=y_{i j} / \sum_{i=1}^{33} y_{i j}$, and suppose that when $f_{i j}=0$, $f_{i j} \ln f_{i j}=0 k=1 / \ln 33$.

Then, the weight $w_{j}$ of the entropy of the jth indicator can be defined as
$w_{j}=\frac{1-H_{j}}{\sum_{j=1}^{7}\left(1-H_{j}\right)}$,

where $0 \leq w_{j} \leq 1$ and $\sum_{j=1}^{7} w_{j}=1$.

A normalized evaluating matrix and the entropy weight are used to calculate the perceived risk index (PRI):

$\mathrm{PRI}_{i}=\sum_{j=1}^{7}\left(w_{j} \times y_{i j}\right)$

A higher PRI indicates a higher perceived risk level.

\subsubsection{Measurement of perceived risk using multiple linear regression}

Multiple linear regression is used in this research to quantitatively measure the changes in the PRI under different driving conditions. In the regression model, weather condition and road type are taken as arguments, and the PRI is taken as dependent variable. Due to the discrete features of the arguments, the two arguments are transformed into two and ten binary variables (taking values of 0 or 1), respectively. Interaction influence is not considered because the weather conditions and road types have no significant interaction effects on the indicators, as shown in Table 3.

The regression results are shown below, and the residual plot is shown in Fig. 6.

$$
\begin{aligned}
& \mathrm{PRI}=0.461-0.112 x_{\text {Uphill }}-0.217 x_{\text {Downhill }}-0.010 x_{\mathrm{LF}} \\
& \quad+0.075 x_{\mathrm{F}}+0.110 x_{\mathrm{DF}}+0.017 x_{\mathrm{HDF}}-0.034 x_{\mathrm{LR}} \\
& \quad+0.1756 x_{\mathrm{R}}+0.156 x_{\mathrm{HR}}+0.146 x_{\mathrm{EHR}}+0.149 x_{\mathrm{S}} \\
& \quad+0.318 x_{\mathrm{EHS}}, R^{2}=0.924
\end{aligned}
$$

where arguments, e.g., $x_{\mathrm{Uphill}}, x_{\text {Downhill }}$ and $x_{\mathrm{LF}}$, are the binary variables for road type and weather condition.

As the $R$-square is high enough and the residual plot shows an unbiased and homoscedastic distribution, the results of linear regression is thought to be valid.

The values of the coefficient represent the influence of factors on the PRI. The reference values are the ones of basic segment and clear sky, which are implied in the constant; their influence value is zero. The coefficients of weather condition and road type are plotted in Fig. 7.

\subsubsection{Influence of road type}

Figure 7 shows that the perceived risk when driving on slopes is lower than those when driving on the basic segment, as illustrated by their negative coefficients. This result may be caused by large headway distance and high 


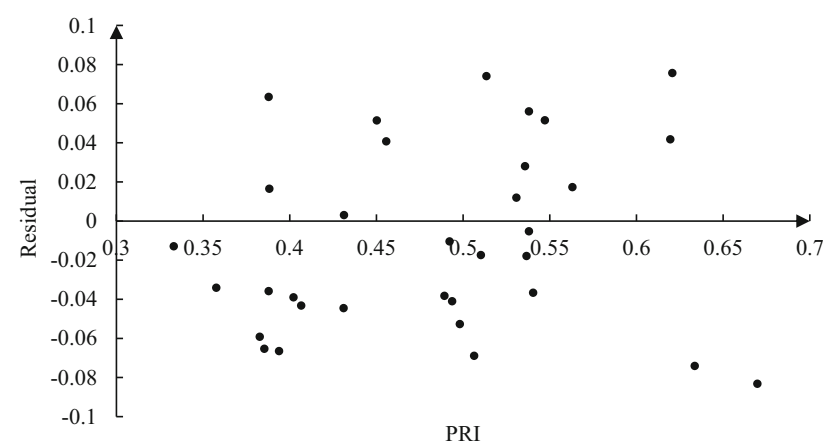

Fig. 6 Residual plot of PRI

absolute value of NA when driving on the basic segment, as shown in Fig. 5.

\subsubsection{Influence of weather condition}

Light fog and light rain have a minor influence on the perceived risks, as illustrated by their small coefficients (nearly zero).

For fog conditions, with the exception of HDF, perceived risk levels increase with fog intensity due to the reduction in visibility. The exception for HDF may be attributed to the extremely poor visibility, which influences the car-following behavior considerably, and leads to a small headway such that the lead car can stay in sight.

With regard to the rain conditions, rain, heavy rain and extremely heavy rain have close coefficients, which means that the perceived risk in rainy days will not increase with rain intensity after it approaches a limit value.

For snow weather conditions, the perceived risk level increases with rain and snow intensity. In particular, extremely heavy snow greatly increases the perceived risk level for drivers due to the low road surface friction.

The model can also be regarded as a prediction model to obtain the PRI before adverse weather occurs, which may help develop traffic management strategies to improve the cautiousness of drivers and further reduce the number of

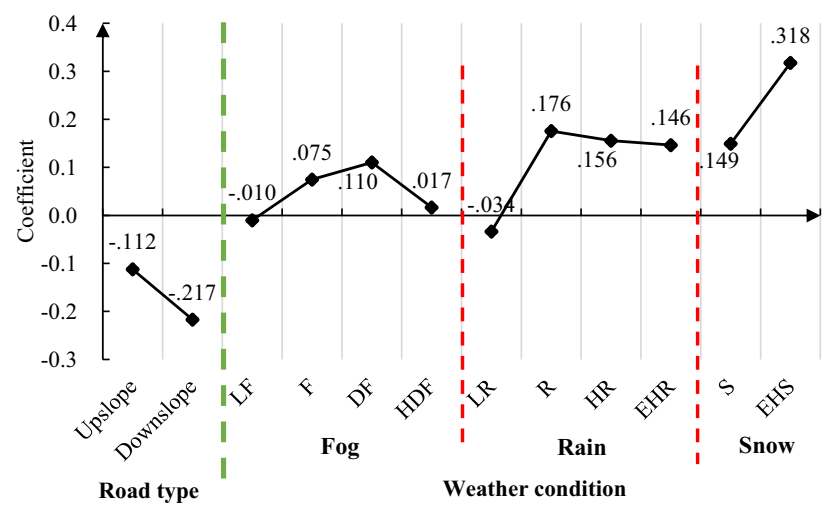

Fig. 7 Coefficients of weather condition and road type accidents. In Fig. 7, the PRI value in heavy dense fog is low, which indicates that specific countermeasures should be taken if the HDF weather is forecasted. Besides, PRI values of both the upslope and downslope are lower than that of basic segment, which suggests that drivers need to pay more attention to their manipulations when driving on slopes.

\section{Discussion}

Due to the limitations in the road friction settings in the driving simulation software, four/two levels of rain/snow weather conditions are considered in this work. The advantage of driving simulator experiment is that it can control experimental conditions. In our experiment, the speed of lead car and surrounding environment are fully controlled by driving simulator software and only weather condition varies. Besides, when conducting the experiment, external factors such as the experiment time, and drivers' mental state are also fully controlled. Compared with the field data research, this experiment can obtain drivers' perceived risk in extreme weather conditions and then help managers to take special countermeasures to reduce crash risk.

In this simulation experiment, the single speed design was used due to the fact that traffic is typically slow under adverse weather conditions. In future work, other traffic situations will be considered to further understand the impacts of adverse weather on drivers' perceived risk.

In Fig. 3, the road surface during snow is black instead of white. This is because the simulator cannot display the scene of snow on pavement. This is one of shortcomings in our snow scenario. However, the visual effect of snowfall looks desirable and the friction of road surface also changed. Actually, the snow on pavement in cities like Beijing will be cleared soon. So participants did not pay attention to this shortcoming. In this sense, this scenario can be used to analyze the influence of snow on driver behaviors.

TTC, an indicator widely used in previous driving risk literature, is not considered in this paper. The reason is that the speed of the lead car is fully controlled by the driving software, which is low and consistent in our driving simulation experiment in most of the time and only changes in acceleration or deceleration car-following situation. Besides, drivers follow the lead car within a close distance and the acceleration values of lead car is very small (3.33 or $-3.33 \mathrm{~km} / \mathrm{h}$ ). Thus, the distance is thought to be a more direct measure for drivers to assess their risk. Therefore, headway or time headway is more appropriate when considering the perceived risk in the experiment than TTC.

In terms of the influence of road type on perceived risk for drivers, the results demonstrate that drivers perceive 
low risk on slopes. This is difficult to explain but it suggests that the difficulty of vehicle operation on slopes makes the vehicle follow the lead car closely and needs large accelerations, thus giving rise to a lower PRI. Slope grade may be an important influential factor; however, only one level is considered in our study $(1.5 \%)$. Unlike road type, weather conditions affect the perceived risk in a different way, and further analyses should be performed to study the difference.

\section{Conclusions}

This work quantitatively describes the influence of adverse weather on the perceived risk for drivers during car following based on a driving simulation experiment. Weather condition and road type have been taken as two factors. Several driving behavior indicators related to risk are selected based on risk homeostasis theory, and the driver's PRI is proposed and calculated using the entropy weight method. Then, multiple linear regression is introduced to measure the effects of both weather conditions and road type on the PRI.

This work allows the drivers' perceived risk level to be determined in advance. In addition, together with the data from road accidents, it can help develop proper strategies to improve driver cautiousness and further reduce the number of road crashes.

The conclusions are summarized as follows:

- Both weather condition and road type have significant impacts on most of the driving behavior indicators. No interaction influence between these indicators is observed.

- Low-intensity fog and rain have a minor influence on drivers' perceived risk, whereas the snow has a significant effect on perceived risk.

- The drivers' perceived risk increases with a deterioration in the weather conditions. The drivers' perceived risk decreases under the conditions of extremely poor visibility, such as HDF.

- Specific countermeasures should be taken in heavy dense fog weathers.

- Drivers need to pay more attention to their manipulations when driving on slopes.

Acknowledgements This study was supported by the National Natural Science Foundation of China project (61672067), and Science and Technology Program of Beijing (Z151100002115040).

Open Access This article is distributed under the terms of the Creative Commons Attribution 4.0 International License (http:// creativecommons.org/licenses/by/4.0/), which permits unrestricted use, distribution, and reproduction in any medium, provided you give appropriate credit to the original author(s) and the source, provide a link to the Creative Commons license, and indicate if changes were made.

\section{References}

1. Rahman A, Lownes NE (2012) Analysis of rainfall impacts on platooned vehicle spacing and speed. Transp Res F Traffic Psychol Behav 15(2):395-403

2. Billot R, El Faouzi NE, De Vuyst F (2009) Multilevel assessment of the impact of rain on drivers' behavior: standardized methodology and empirical analysis. Transportation Research Record: Journal of the Transportation Research Board, No. 2017, pp 134-142

3. Saberi M, Bertini RL (2010) Empirical analysis of the effects of rain on measured freeway traffic parameters. Transportation research board 89th annual meeting. No. 10-2331

4. Yan X, Li X, Liu Y, Zhao J (2014) Effects of foggy conditions on drivers' speed control behaviors at different risk levels. Saf Sci 68:275-287

5. Konstantopoulos P, Chapman P, Crundall D (2010) Driver's visual attention as a function of driving experience and visibility. Using a driving simulator to explore drivers' eye movements in day, night and rain driving. Accid Anal Prev 42(3):827-834

6. Broughton KLM, Switzer F, Scott D (2007) Car following decisions under three visibility conditions and two speeds tested with a driving simulator. Accid Anal Prev 39(1):106-116

7. Yamaguchi M, Sakakima J (2007) Evaluation of driver stress in a motor-vehicle driving simulator using a biochemical marker. J Int Med Res 35(1):91-100

8. Rhodes N, Pivik K (2011) Age and gender differences in risky driving: the roles of positive affect and risk perception. Accid Anal Prev 43(3):923-931

9. DeJoy DM (1991) An examination of gender differences in traffic accident risk perception. Accid Anal Prev 24(3):237-246

10. Bella F (2013) Driver perception of roadside configurations on two-lane rural roads: effects on speed and lateral placement. Accid Anal Prev 50:251-262

11. Hjelkrem OA, Ryeng EO (2016) Chosen risk level during carfollowing in adverse weather conditions. Accid Anal Prev 95:227-235

12. Summala H (1996) Accident risk and driver behaviour. Saf Sci 22(1):103-117

13. Wilde GJS (1982) The theory of risk homeostasis: implications for safety and health. Risk Anal 2(4):209-225

14. Ding H, Zhao X, Rong J, Ma J (2013) Experimental research on the effectiveness of speed reduction markings based on driving simulation: a case study. Accid Anal Prev 60(2013):211-218

15. Zhao X, Guan W, Liu X (2013) A pilot study verifying how the curve information impacts on the driver performance with cognition model. Discrete Dyn Nat Soc 2013:316896

16. China Meteorological Administration (2019) Highway traffic meteorological condition levels. http://www.cma.gov.cn/2011wmhd/ 2011wzqyj/201111/P020111115369349788700.doc. Accessed 28 Aug 2019

17. China Meteorological Administration (2019) Rainfall levels. http:// www.cma.gov.cn/2011xzt/2013zhuant/20130620_3/2013062002/ 201308/t20130816_223400.html. Accessed 28 Aug 2019

18. China Meteorological Administration (2019) Conditions that forming snow and classification. http://www.cma.gov.cn/2011xzt/ kpbd/SnowStorm/2018050902/201811/t20181106_482641.html. Accessed 28 Aug 2019 